Received: May 03, 2017 Accepted: May 13, 2017 Published: May 23, 2017

\title{
Controversies in Pregnancy Management after Prenatal Diagnosis of a Twin Pregnancy Discordant for Trisomy 21 Diagnosed by Cell-Free Fetal DNA Testing
}

\author{
Emine Cetin ${ }^{1}$, Tamina Rawnaq ${ }^{2}$, Boris Schulze-Koenig ${ }^{1}$, Moritz Doebert ${ }^{2}$, Panagiota Zoi $^{3}$ \\ and Peter Schwaerzler ${ }^{2 *}$ \\ ${ }^{1}$ Center for Prenatal Diagnosis and Fetal Genetics Hamburg, Altonaer Straße 61, 20357 \\ Hamburg, Germany. \\ ${ }^{2}$ Department of Obstetrics and Gynecology Asklepios Clinic Barmbek, Ruebenkamp 220, \\ 22307 Hamburg, Germany. \\ ${ }^{3}$ Asklepios Medical School, Pro-research, Lohmuehlenstraße 5, 20099 Hamburg, \\ Germany.
}

*Corresponding author: Prof. Dr. Peter Schwaerzler, Asklepios Clinic Barmbek, Department of Obstetrics and Gynecology, Ruebenkamp 220, 22291-Hamburg, Germany. Tel: +49401818821840 / +4915125337260;Fax: +49401818821849;:E-mail: p.schwaerzler@asklepios.com

\section{Abstract}

\subsection{Background}

Current preliminary data is advocating cfDNA testing in twin pregnancies since both increasing use of ART and higher maternal ages have raised the incidence of (discordant) aneuploidies.

\subsection{Procedures and findings}

This report is raising ethical implications deriving from a twin pregnancy discordant for trisomy 21 conceived from egg donation and diagnosed by cfDNA testing after low risk conventional first-trimester screening.

\subsection{Conclusion}

The solution of the consequent pregnancy conflict was achieved by adoption of the affected twin instead of selective fetocide.

\subsection{Keywords}

Twin pregnancy, cfDNA testing, fetal aneuploidy, late termination of pregnancy, selective fetocide

\section{Introduction}

The detection rates of currently implemented prenatal screening methods for trisomy 21 based on an algorithm of both sonographic and biochemical markers are - in the best care - 90-
$95 \%$ with a false-positive rate of $3-5 \%$, hence, still entailing a procedure related miscarriage rate of approximately $1 \%$. The realization that fetal nucleic acids are present in maternal blood has spawned efforts to analyze cell free (cf) DNA amongst others for fetal aneuploidy detection. The chromosomal dosage resulting from fetal aneuploidy is directly related to the fraction of fetal cfDNA [1]. We report on prenatal diagnosis of a dichorionic twin pregnancy discordant for trisomy 21 by cfDNA testing following low risk estimation by a conventional first trimester screening (FTS).

\section{Methods and Results}

This is the remarkable story of a 50 year-old woman in her sixth pregnancy after having had egg donation from a 27-year-old donor and intracytoplasmic sperm injection (ICSI) resulting in a dichorionic twin pregnancy. She has had three uneventful pregnancies and two miscarriages in her history and requested FTS at 13 weeks of gestation. Nuchal translucency (NT) measurement showed discordance with 2,0 and $3,4 \mathrm{~mm}$ in fetus 1 and fetus 2 , respectively (Fig 1). However, combined test including nasal bone, tricuspid valve regurgitation and ductus venosus pulsatility index (PI) according to first-trimester contingent screening for trisomy 21 gave a risk of 1:17974 and 1.1300, respectively. Detailed survey of the risk calculation is given in table 1. After extensive genetic counseling, the couple declined any further non-invasive or invasive testing. A follow-up scan at 17 weeks of gestation additionally revealed an echogenic focus in the left cardiac ventricle of fetus 2 prompting the couple to request a second stage test and cfDNA testing was offered in a contingent model [2]. The Harmony ${ }^{\mathrm{TM}}$ Prenatal Test (Ariosa Diagnostics, Inc., San Jose, CA, USA) using the smallest fetal fraction (FF) contribution 
Table 1 Background and adjusted risks of both fetuses for the trisomies 21,18 and 13

\begin{tabular}{|l|l|l|l|}
\hline & Trisomy 21 & Trisomy 18 & Trisomy 13 \\
\hline $\begin{array}{l}\text { Background } \\
\text { risk }\end{array}$ & 0.665972222 & 1.615277778 & 4.964583333 \\
\hline $\begin{array}{l}\text { Adjusted risk } \\
\text { fetus 1 }\end{array}$ & $1: 17974$ & $1: 45321$ & $1: 15313$ \\
\hline $\begin{array}{l}\text { Adjusted risk } \\
\text { fetus 2 }\end{array}$ & 0.949305556 & 2.455555556 & 0.661805556 \\
\hline
\end{tabular}

Fetus 1 had a Nuchal translucency of $2.0 \mathrm{~mm}$ and an adjusted risk of $<1: 17974$ for trisomy 21 .

Fetus 2 had anadjusted risk of 1:1300 following risk calculation using the algorithm of the FMF London.

The adjusted risk for trisomy 13 amounted to 1:893 in fetus

2 due to an increased heart rate of 175 beats per minute

of the 2 fetuses for its FF optimized risk of trisomy evaluation $\left(\right.$ FORTE $^{\mathrm{TM}}$ ) algorithm was carried out and risk scores for each trisomy were provided as a percentage with ranges capped at $>99 \%$ and $<0.01 \%$ [3]. The first sample did not yield a result due to low FF and a second sample taken at 20 gestational weeks reported a high risk for trisomy 21 (smallest FF contribution 4.0\%) in one of the 2 fetuses [4] that was confirmed for fetus 2 by rapid quantitative fluorescent polymerase chain reaction (QF-PCR) and subsequent conventional karyotyping. Detailed anomaly scan revealed no cardiac defects or other major structural anomalies in both of the twins, nevertheless, the couple and in particular the husband demanded selective fetocide [5] of the affected fetus referring to section 218a of the German panel code.

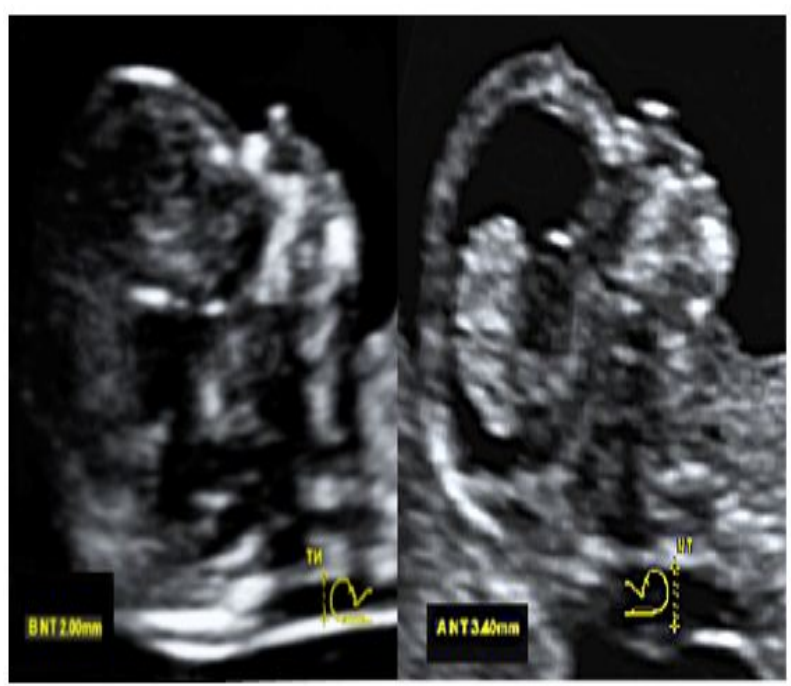

Figure 1:Measurement of Nuchal translucency in both fetuses. Measurement of Nuchal translucency in both fetuses at $12+5$ weeks according to the guidelines of the Fetal Medicine Foundation (FMF). Fetus 1 and 2 had a Nuchal translucency (NT) of 2.0 $\mathrm{mm}$ and $3,4 \mathrm{~mm}$, giving an adjusted risk of $1: 17974$ and $1: 1348$, respectively, for trisomy 21 .

To approach the complex and conflicting situation an appeal to the local ethics committee was suggested and the couple's various aspects of the social and familial relationships, the scope and limits of the test results as well as alternative pregnancy management options were discussed. The committee unanimously opposed to selective fetocide and proposed placement of the trisomic baby for adoption after delivery. Supported by dedicated midwifes and professional psychological counseling the couple decided to continue the twin pregnancy giving consent to adoption of the trisomic newborn immediately after delivery. Ultrasound follow-up scans performed every two weeks revealed no additional anomalies, however, the growth measurement in both fetuses were slowing down and uneventful primary Caesarean section at $36+5$ weeks of gestation, three days before Christmas, was indicated. The non-affected boy, (2790 g, APGAR score 9/9/9, umbilical artery ph 7.26) stayed with the parents. The trisomic boy (2190 g, APGAR score 8/9/10, umbilical artery ph 7.26) was welcomed by his new family after 28 days of hospitalization.

\section{Discussion}

From our perspective this case is raising issues both of medical and genetic nature and of controversial ethical and social aspects relevant to that providing contemporary pregnancy care.

1. What is the professional information and counseling for a 49 year-old woman who already has three grown-up children and now has a new partner, 14 years younger, when she desires another pregnancy?

2. Why did the best contemporary ultrasound-based screening strategy currently available fail to detect discordant fetal aneuploidy in a twin pregnancy at 11-14 weeks?

3. What is the indication for cfDNA testing in twin pregnancies and why was there a low FF at the first attempt of cfDNA testing at 17 weeks delaying the diagnosis beyond 20 weeks of pregnancy? 4. What are the implications and management options emerging from prenatal diagnosis of a twin pregnancy discordant for trisomy 21 at 22 weeks of pregnancy after having taken the effort of a substantial medical and financial burden to get pregnant before?

Rapid advances in reproductive medicine have helped many couples to overcome personal and family related obstacles, which prevented them from having tailored life planning. However, from our perspective it is crucial to provide pre-treatment counseling by addressing the issue of transferring more than one embryo and focusing in particular on the ethical conflict entailing the diagnosis of discordant aneuploidies in multiple pregnancies before embarking on ART. The issue of late termination following treatment by ART in general and selective fetocide in particular imposes a huge challenge to both the affected couple and the involved fetal medicine specialists. The impossibility of finding "good" solutions by addressing the needs of the future parents and the rights of the unborn fetuses is creating measures of prevention e.g. In form of single embryo transfer of utmost relevance.

In the last decade various, mainly ultrasound based screening strategies, have brought pregnancy care to a level never seen before in many countries worldwide. However, with regard to fetal aneuploidies the sensitivity and specificity of recently available cfDNA testing has proven to be superior to that of FTS. In single- 
tons data from the Next study suggest that up to 1 in 5 fetuses with Down syndrome can go undetected on FTS [6]. Several clinical studies in high-risk pregnancies and a recent study in a population undergoing routine first-trimester aneuploidy screening have demonstrated that the performance of non-invasive prenatal testing (NIPT) for trisomy 21 , with a detection rate of $>99 \%$ and false-positive rate of $<0.1 \%$, is far superior to that of all other currently available screening methods for Down syndrome [6]. Moreover, recent meta-analysis data suggest performance of FTS in twins being inferior to that in singletons, so the false negative rate could be even higher in dichorionic twins accounting for a sensitivity of only $86 \%$ [7]. Our case in accordance with recent data is supporting the role of cfDNA testing in screening for trisomy 21 in twins.

A potential issue with NIPT as a universal screening test is the failure rate in providing a result, which primarily depends on the relative proportion of fetal to maternal origin of the cfDNA in maternal plasma [8]. In trisomic pregnancies DNA derived from the extra fetal chromosome results in a higher proportion of fetal DNA than in disomic pregnancies. The ability to detect this small increase in the amount of a given chromosome in maternal plasma in a trisomic pregnancy is directly related to the FF. However, if the FF is below 4\%, NIPT fails to provide a result [9], thus - as happened in our case - delaying the diagnosis well into the second trimester. Several predetermining factors such as maternal BMI of 38, advanced maternal age (50) and ARTlinked impaired placentation [10] may explain the surprisingly low FF at the first attempt of cfDNA testing at 17 weeks of pregnancy. From our experience the estimation of the lower FF is what matters clinically, because the potential source of an erroneous result from cfDNA screening for aneuploidies in dizygotic twins is failure to recognize the cases with a satisfactory total FF but with an unsatisfactory lower FF for 1 fetus of less than 4\%. Fetal aneuploidy assessment with cfDNA analysis has proven to be clinically useful in singleton pregnancies. While twin pregnancies pose some additional complexity, it is likely that cfDNA analysis - as shown in our case - will also be clinically useful in this population. However, the proposed approach for cfDNA testing in monozygotic and dizygotic twins requires further investigation in clinical implementation studies. Performing selective fetocide at 22 weeks of gestation entails an increased risk of morbidity and mortality to the not affected co-twin ranging from 5-15\% depending on the phase of pregnancy the intervention is performed [5]. Moreover, doing this intervention to a fetus with Down syndrome at 22 weeks of pregnancy revealing - apart from soft markers no obvious abnormalities on detailed anomaly scan was thought not only to be ethically questionable but also being a potentially traumatic experience to both the couple involved and the fetal medicine specialist performing the procedure. So we brought up the idea of adoption instead of fetocide and after extensive counseling and supported by dedicated staff the couple opted for this option. We are aware that this option is not a perfect solution for this pregnancy conflict but in this situation there is no good solution. The effort can only be to find the least traumatic and unkind way to cope with this situation for everybody involved. In our experience the option of adoption proved to be a bearable way in this complex situation and to many members of the ethics committee and other involved staff it was a moving surprise how easy it was to find parents happily ready to accept and foster a newborn Down syndrome baby.

\section{References}

1. Sparks AB, Struble CA, Wang ET, Song K, Oliphant A. Noninvasive prenatal detection and selective analysis of cell-free DNA obtained from maternal blood: evaluation for trisomy 21 and trisomy 18. Am J Obstet Gynecol. 2012; 206(4): 319. e1-9. Doi 10.1016/j.jog.2012.01.030

2. Nicolaides KH, Wright D, Poon LC, Syngelaki A, Gil MM. First-trimester contingent screening for trisomy 21 by biomarkers and maternal blood cell-free DNA testing. Ultrasound Obstet Gynecol. 2013;42(1):41-50. Doi: 10.1002/uog.12511

3. Sparks AB, Wang ET, Struble CA, Barrett W, Stokowski $\mathrm{R}$, mcbride $\mathrm{C}$, et. Al. Selective analysis of cell-free DNA in maternal blood for evaluation of fetal trisomy. Prenat Diagn. 2012;32(1):3-9. Doi: 10.1002/pd.2922

4. Bevilacqua E, Gil MM, Nicolaides $\mathrm{KH}$, Ordonez E, Cirigliano V, Dierickx H, et al. Performance of screening for aneuploidies by cell-free DNA analysis of maternal blood in twin pregnancies. Ultrasound Obstet Gynecol. 2015; 45(1):61-66. Doi: 10.1002/uog. 14690

5. Evans MI, Goldberg JD, Dommergues M, Wapner RJ, Lynch L, Dock BS, et al. Efficacy of second-trimester selective termination for fetal abnormalities: international collaborative experience among the world's largest centers. Am J Obstet Gynecol. 1994; 171(1):90-94

6. Norton ME, Jacobsson B, Swamy GK, Laurent LC, Ranzini AC, Brar $\mathrm{H}$, et al. Cell-free DNA analysis for noninvasive examination of trisomy. N Engl J Med. 2015;372(17):1589-1597. Doi: 10.1056/nejmoa140739

7. Prats P, Rodriguez I, Comas C, Puerto B. Systematic review of screening for trisomy 21 in twin pregnancies in first trimester combining nuchal translucency and biochemical markers: a meta-analysis. Prenat Diagn. 2014;34(11): 1077-1083. Doi: 10.1022/pd.4431

8. Verweij EJ, van den Oever JM, de Boer MA, Boon EM, Oepkes D. Diagnostic accuracy of noninvasive detection of fetal trisomy 21 in maternal blood: a systematic review. Fetal Diagn Ther. 2012;31(2):81-86. Doi: 10.1159/000333060

9. Brar H, Wang E, Struble C, Musci TJ, Norton ME. The fetal fraction of cell-free DNA in maternal plasma is not affected by a priori risk of fetal trisomy. J Matern Fetal Neonatal Med. 2013;26(2):143-145. Doi: 10.3109/14767058.2012.722731

10. Ashoor G, Syngelaki A, Poon LC, Rezende JC, Nicolaides $\mathrm{KH}$. Fetal fraction in maternal plasma cell-free DNA at 11-13 weeks' gestation: relation to maternal and fetal characteristics. Ultrasound Obstet Gynecol. 2013;41(1):26-32. Doi: 10.1002/uog.12331 